\title{
The natural history of community-acquired common colds symptoms assessed over 4-years*
}

\author{
Theodore J. Witek', David L. Ramsey², Andrew N. Carr², Donald K. Riker ${ }^{3}$ \\ ' Healthcare Innovation and Technology Laboratory at Columbia University in The City of New York, NY 10032 USA \\ 2 Procter \& Gamble, Personal Health Care Division, Mason, OH 45040, USA \\ ${ }^{3}$ On Point Advisors, LLC, PO Box 5450, Chattanooga, TN 37406, USA
}

Rhinology 53: 81-88, 2015

DOl:10.4193/Rhino14.149

*Received for publication:

June 18,2014

Accepted: September 7, 2014

\begin{abstract}
Background: The common cold is the most frequently experienced infection among humans, but limited data exist to characterize the onset, duration, severity and intersection of symptoms in community-acquired colds. A more complete understanding of the symptom frequency and burden in naturally occurring colds is needed.
\end{abstract}

Methodology: We characterized common cold symptoms from 226 cold episodes experienced by 104 male or female subjects. Subjects were enrolled in the work environment in an attempt to start symptom evaluation (frequency and severity) at the earliest sign of their cold. We also assessed the symptom that had the greatest impact on the subject by asking them to identify their single most bothersome symptom.

Results: Symptom reporting started within 24 hours of cold onset for most subjects. Sore throat was a harbinger of the illness but was accompanied by multiple symptoms, including nasal congestion, runny nose and headache. Cough was not usually the most frequent symptom, but was present throughout the cold, becoming most bothersome later in the cold. Nasal congestion, pain (eg, sore throat, headache, muscle pains) or feverishness and secretory symptoms (eg, runny nose, sneezing), and even cough, were simultaneously experienced with high incidence over the first 4 days of illness. The single most bothersome symptom was sore throat on day 1, followed by nasal congestion on days 2-5 and cough on days 6 and 7 .

Conclusion: There is substantial overlap in the appearance of common cold symptoms over the first several days of the common cold. Nasal congestion, secretory and pain symptoms frequently occur together, with cough being somewhat less prominent, but quite bothersome when present. These data establish the typical symptomatology of a common cold and provide a foundation for the rational treatment of cold symptoms typically experienced by cold sufferers.

Key words: common cold, acute upper respiratory tract infection, URTI, nasal congestion, common cold symptoms, rhinovirus, cold viruses, natural history, bothersomeness

\section{Introduction}

The common cold is a ubiquitous, but self-limited, viral illness responsible for the global loss of productivity ${ }^{(1)}$, high healthcare costs ${ }^{(2)}$ and a sometimes prodrome, or trigger of, other illnesses ${ }^{(3)}$, for example asthma. Viral illness has been suggested to underlie $50 \%$ of all illness in adults and $75 \%$ in children ${ }^{(4)}$. The cause of an acute URTI or common cold (or simply "cold") is due to infection by one or more of over 200 known viruses, typically a rhinovirus ( $30-50 \%)$, but can also be due to coronavirus (10-
$15 \%)$, influenza or "flu" (5-15\%), adenovirus (<5\%), parainfluenza virus $(\sim 5 \%)$, respiratory syncytial virus $(\sim 5 \%)$ and enterovirus $(<5 \%)^{(5)}$. These viruses can infect the tissues of the upper and/or lower airways with symptoms ranging from local irritation and inflammation to systemic body aches and pains. Although there is no cure for the common cold, the individual and grouped symptoms of the common cold drive consumers to seek palliative relief, largely from over-the-counter products to mitigate the impact of symptoms, and to maintain patient performance 
and quality of life ${ }^{(6,7)}$. The temporal evolution of infection and inflammation following respiratory exposure to colds viruses have been documented ${ }^{(3,8,9)}$. Moreover, the progression and evolution of symptoms during community-acquired colds have been described using subjective symptom rating systems in all sufferers ${ }^{(10,11)}$ and in rhinovirus-positive sufferers ${ }^{(12-14)}$, as well as described using quality of life instruments ${ }^{(15)}$. To this end, as early as 1958, Jackson et al. described the time course of symptoms in healthy volunteers with experimental colds, establishing 8 core common cold symptoms as sneezing, headache, malaise, chilliness, nasal discharge, nasal obstruction, sore throat and cough ${ }^{(16)}$. In this seminal publication, headache, sneezing, chilliness and sore throat were considered early symptoms while nasal discharge, nasal obstruction, malaise and cough were later, more persistent symptoms. A study by Gwaltney in 1967 detailed the symptoms of the common cold in the work environment over 22 months and focused on differences between those colds identifiable as rhinovirus-positive. Overall this study identified similar symptoms, but did not reproduce the time course of symptoms described by Jackson ${ }^{(16)}$.

While the experience of a cold is common to everyone, much of the data attempting to describe the earliest stage of the illness describe symptomatology after inoculation of a laboratory passaged rhinovirus strain into the nasal airway. The phenotypic expression of virulence of passaged rhinovirus strains versus prevalent wild types is open to discussion. The study of natural or community-acquired colds is challenging due to difficulties in recruiting subjects in the early part of their cold and is potentially confounded by compensation of subjects (17). To our knowledge, no single prospective investigation has cumulated data on community-acquired colds illness episodes over a 4-year period especially when the majority of subjects were entered within 24 hrs of illness onset.

Therefore, to better describe the evolution of a communityacquired cold's symptoms the objectives of this prospective clinical study were to: 1 ) enter subjects early in the illness; 2 ) assess the temporal occurrence of individual and grouped symptoms, and, 3) determine the "bothersomeness" of symptoms to subjects.

\section{Materials and methods}

\section{Subjects}

This study was a prospective, observational (non-interventional) study designed to initiate surveillance as soon as an illness episode began in healthy volunteers who were employees of Richardson-Vicks, Inc. (Shelton, CT, USA), then a subsidiary of Procter \& Gamble. The study site was co-located within their place of employment. The study evaluated 226 cold episodes in 104 male and female subjects over a 4-year time period.
Subjects were eligible to participate in the study if they were in good health as assessed by medical history, gave informed consent, and were at least 18 years old. Subjects were excluded from participating if they had evidence of an upper respiratory tract infection (URTI) or fever within one week of enrollment, had a history of atopic dermatitis, deviated septum, nasal polyps, sinusitis, asthma, chronic rhinitis, or other chronic respiratory condition, used any prescription medication within one week of symptom evaluation that may impact symptoms, used any OTC medication within 48 hours of the screening visit, or used any investigational drug within 1 month of screening, or admitted to use of licit or illicit nasal drugs.

Subjects were enrolled and instructed to report to the study site at the first sign of a common cold illness. Subjects were excluded from further evaluation if the cold was more than 48 hours old or if the first day they reported the cold was on a Friday. On Day 1 , subjects' throats were swabbed to determine the presence of Group A beta-hemolytic Streptococcus infection. Those testing positive for Streptococcus infection were referred to the study physician and their current cold episode was not further evaluated. Subjects reported cold symptoms they were experiencing at the moment of query and rated overall severity of their cold on a $0-4$ scale $(0=$ not present, $1=$ mild, $2=$ moderate, $3=$ severe, 4 $=$ very severe). Subjects were asked if they had taken any OTC or prescription medications in the past 24-48 hours, or prescription medication in the past week. Subjects reported the number of hours since their first cold symptom. To be considered a cold episode and for the data to be evaluated, the symptoms must have lasted more than 1 day. While only cold episodes reported with symptom onset of $\leq 48$ hours were entered and evaluated, $87 \%$ entered the study within 36 hrs of onset and $73 \%$ within 24 hrs. Once a subject was symptom free for 3 consecutive days they were considered eligible for initiation of another illness episode if they met the episode entry criteria.

\section{Assessment of symptoms}

Symptomatology over the first 7 days of the cold was self-assessed in a hardcopy diary asking subjects to assess their current symptoms at 3 pre-determined times a day (9 AM, 3 PM, 9 PM) using a $0-4$ severity scale $(0=$ not present, $1=$ mild, $2=$ moderate, $3=$ severe, $4=$ very severe). During the week, the 9 AM assessment was completed at the study site, while the $3 \mathrm{PM}$ and 9 PM assessments were recorded in the daily symptom diary. Daily symptom assessments on the weekend were assessed at the same 3 time points and recorded in the subject's diary only. Symptoms that were considered by the investigators as pathognomonic of the common cold included: sneezing, runny nose, nasal congestion, sore throat, hoarseness, cough, headache, chilly, malaise and feverishness. Subsequently, additional questions were added to the symptom assessment including night-time cough (evaluated at 9 AM only), post-nasal drip, post- 
Table 1. Demographic characteristics of subjects and estimated time of onset of their colds illness at entry.

\begin{tabular}{|c|c|}
\hline Demographics & Cold sufferers $(n=104)$ \\
\hline \multicolumn{2}{|l|}{ Age $(n=101)$} \\
\hline Mean (SD) & $32(10.9)$ \\
\hline Min - Max & $18-65$ \\
\hline \multicolumn{2}{|l|}{ Sex } \\
\hline Female & $69(66 \%)$ \\
\hline \multicolumn{2}{|l|}{ Race } \\
\hline Caucasian & $84(81 \%)$ \\
\hline Oriental & $2(2 \%)$ \\
\hline Black & $10(10 \%)$ \\
\hline Hispanic & $5(5 \%)$ \\
\hline Other & $3(3 \%)$ \\
\hline \multicolumn{2}{|l|}{ Baseline characteristics } \\
\hline Allergic rhinitis $(n=101)$ & $12(12 \%)$ \\
\hline Sinusitis, chronic or active & $2(2 \%)$ \\
\hline \multicolumn{2}{|l|}{ Time to report a cold (hrs) } \\
\hline $0-12$ & $28.8 \%(28.8 \%)$ \\
\hline $13-24$ & $44.7 \%(73.5 \%)$ \\
\hline $25-36$ & $13.7 \%(87.2 \%)$ \\
\hline $37-48$ & $12.8 \%(100 \%)$ \\
\hline
\end{tabular}

$\mathrm{N}=$ number of subjects within specified demographic. $\mathrm{n}(\%)=$ number and percentage of subjects within specified parameter, demographic category. Time to report a cold was assessed by asking cold sufferers the "Number of hours since your first cold symptoms" at entry. Cumulative percentages are shown in parentheses.

nasal burning or discomfort, scratchy throat, muscle aches and pains, blocked or "popping" ears, and watery eyes ( $n=138$ colds episodes) and subjects were also asked to report their "one most bothersome symptom" each morning ( $\mathrm{n}=86$ colds episodes). For purposes of this analysis night-time cough and cough, as well as scratchy throat and sore throat were combined. Per the clinical protocol, subjects were "asked to refrain from taking any medication to treat their cold." If medication was prescribed by a physician during the course of the cold, the subject was dropped from the study. All procedures followed in this

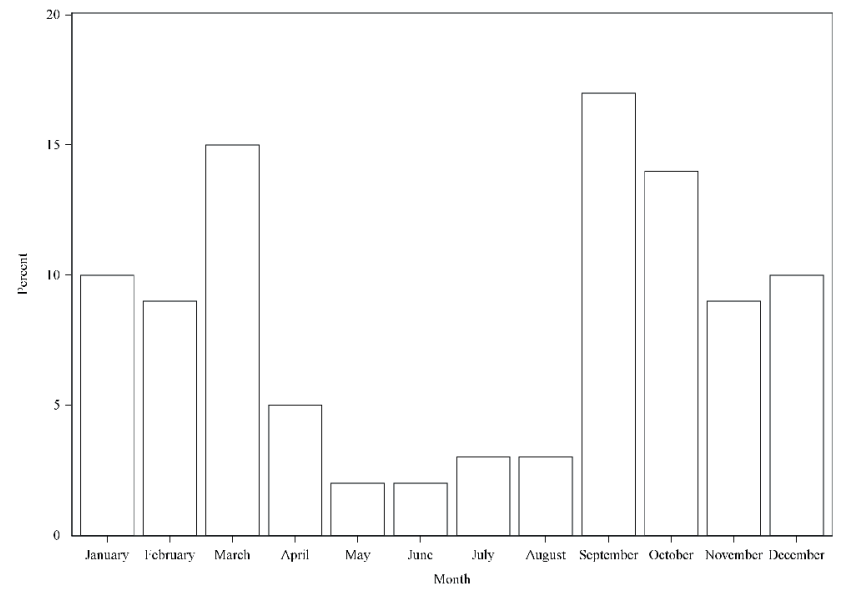

Figure 1. Seasonality of colds illnesses at entry as reported during a calendar year. Percent of cold episodes of greater than 1 day in duration ( $n=226$ episodes) reported by subjects for each month over a 4 year period.

surveillance were in accordance with the ethical standards and approval of our Institutional Review Board (Product Investigations, Inc. Coshohocken, PA, USA) and with the Helsinki Declaration (1964). All subjects granted written informed consent to survey their colds illnesses. No patient was administered treatment by the sponsor during the course of this study.

\section{Results}

Over the course of 4 years, 156 subjects participated in the study with 104 reporting self-identified colds. A total of 226 cold episodes were reported by these 104 subjects. Demographic data on the subjects are shown in Table 1. The mean age of participants was 32 years of age with females representing $66 \%$ of respondents. The majority of subjects reported no history of allergic rhinitis (88\%), or active/chronic sinusitis (98\%).

The median number of colds reported by subjects was 1 and the maximum number of colds reported by any one subject was 10. To initiate assessment of a cold episode subjects were asked to report the time (in hours) from the onset of symptoms to presentation at the site and most subjects (> 73\%) reported to the site within 24 hours of symptom onset (Table 1). Cold episodes were experienced year round (Figure 1) with the highest percentage of cold episodes occurring in spring and fall months, for example, September (17\%), followed by March (15\%) and October (14\%). The increased occurrence of illness in the spring and fall is consistent with rhinovirus positivity ${ }^{(12)}$.

Subjects were asked to assess the severity of their cold over the first 7 days in the morning (9 AM), afternoon (3 PM), and evening (9 PM), using a 0-4 severity scale (see Methods). On Day 1 , mean cold severity reported by the subjects was 1.8 (moderate), peaked on Day 2 (mean $=1.9$ days) and declined thereafter 

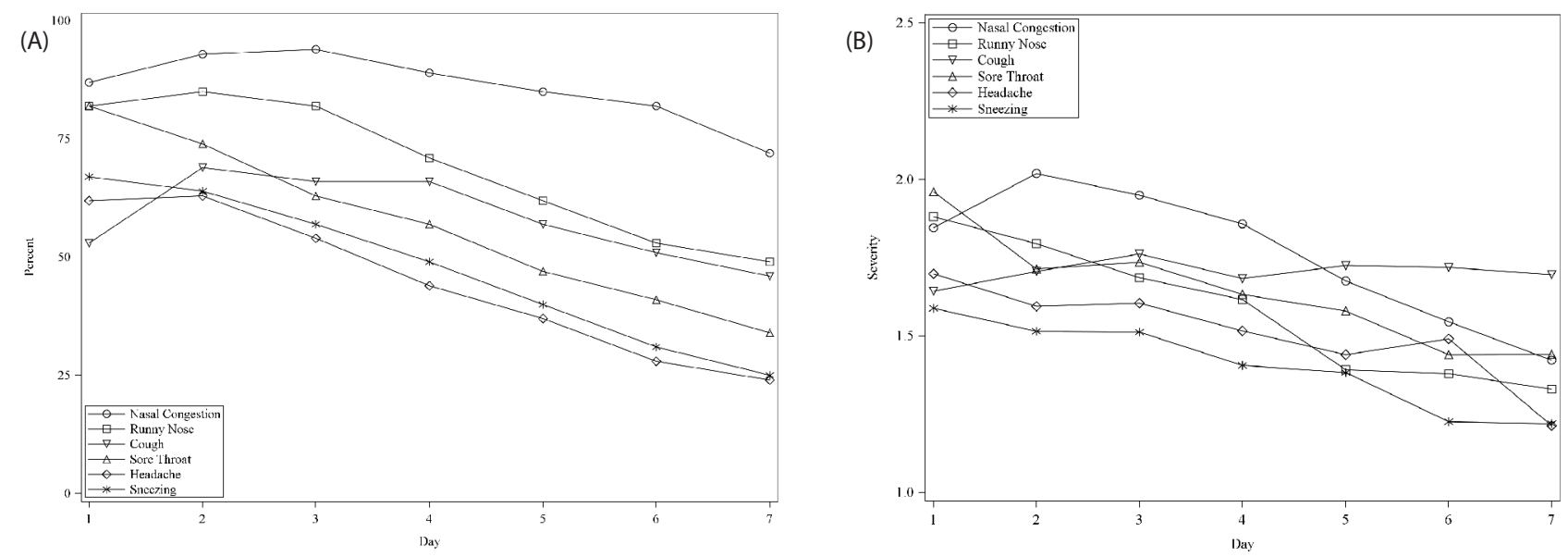

Figure 2. The daily presence and severity of key cold symptoms when assessed at 9 AM. (A) The frequency of common cold symptoms, expressed as a percentage of subjects who self-reported a cold that day (9 AM assessment). (B) The mean severity of common cold symptoms at the 9 AM assessment. Symptom severity was rated on a 5 -point scale $(0=$ not present; $4=$ very severe). Symptoms shown are over the first 7 days of a cold.

Table 2. Daily cold prevalence and symptom severity.

\begin{tabular}{|lccccccc} 
& $\begin{array}{c}\text { Day 1 } \\
(\mathbf{n}=\mathbf{2 2 5})\end{array}$ & $\begin{array}{c}\text { Day 2 } \\
(\mathbf{n}=\mathbf{2 1 7})\end{array}$ & $\begin{array}{c}\text { Day 3 } \\
(\mathbf{n}=\mathbf{2 0 6})\end{array}$ & $\begin{array}{c}\text { Day 4 } \\
(\mathbf{n}=\mathbf{2 0 1})\end{array}$ & $\begin{array}{c}\text { Day 5 } \\
(\mathbf{n}=\mathbf{2 0 2})\end{array}$ & $\begin{array}{c}\text { Day 6 } \\
(\mathbf{n}=\mathbf{2 0 1})\end{array}$ & $\begin{array}{c}\text { Day 7 } \\
(\mathbf{n}=\mathbf{2 0 0})\end{array}$ \\
\hline Symptom prevalence (\%) & 100 & 98 & 95 & 90 & 86 & 81 & 61 \\
\hline Symptom severity (Mean) & 1.8 & 1.9 & 1.8 & 1.7 & 1.5 & 1.4 & 1.4
\end{tabular}

Daily prevalence of a cold reflects a subject's answer to the question "Do you have a cold today?" when asked each morning at 9 AM. Cold symptom severity is the daily mean total symptom score reported by those cold sufferers reporting that they had a cold. The severity scale was 0-4 (see Methods).

(Table 2). On Day 7, 61\% of subjects indicated that they were still suffering from at least one cold symptom, although the mean severity (mean $=1.4$ ) was decreased compared to Day 1 .

Previous studies examining symptoms in rhinovirus-induced cold models have described variation in symptoms over the course of the day ${ }^{(18)}$. Comparing the assessments at 9 AM, 3 PM and 9 PM, symptom frequency and severity decreased throughout the day and across the week with no clear temporal patterns (data not shown). Given this finding and for simplicity, all individual symptom data are presented from the 9 AM on-site assessment. Nasal congestion was the predominant symptom of most cold episodes, peaking on Day 3 (reported in $94 \%$ of cold episodes) with $\geq 85 \%$ of cold episodes characterized by the presence of this symptom throughout the day for the first 5 days (Figure 2A). The symptoms of sore throat and sneezing were most prominent on Day 1 with symptoms reported in $82 \%$ and $67 \%$ of cold episodes, respectively. Runny nose (85\%) and headache (63\%) peaked on Day 2. Each of these symptoms stea- dily declined in frequency and severity towards Day 7. Occurrence of cough, on the other hand, peaked at Day 2 (69\%) and remained high until Day 4 (66\%), with a slow decline thereafter.

Symptom severity data (mean severity at 9 AM) over the course of the first 7 days of a cold (Figure 2B) was generally maintained over the course of a cold with higher severity reported on Days 1 and 2 for most symptoms. The most severe symptom on Day 1 was sore throat, (reported as at least moderate in $56 \%$ of cold episodes), followed by nasal congestion on Days 2-4 and cough on Days 5-7. While runny nose was never reported as the most severe symptom, it was described as at least moderate in $52 \%, 53 \%$ and $40 \%$ of cold episodes for the first 3 days. Other symptoms, including headache and sneezing were generally not as severe as sore throat, nasal congestion, cough and runny nose (Figure 2B) but were described as at least of moderate severity on Day 1 in 34\% and 32\% of colds, respectively. In an effort to assess which cold symptom was most impactful, we asked cold sufferers to indicate which single cold symptom 


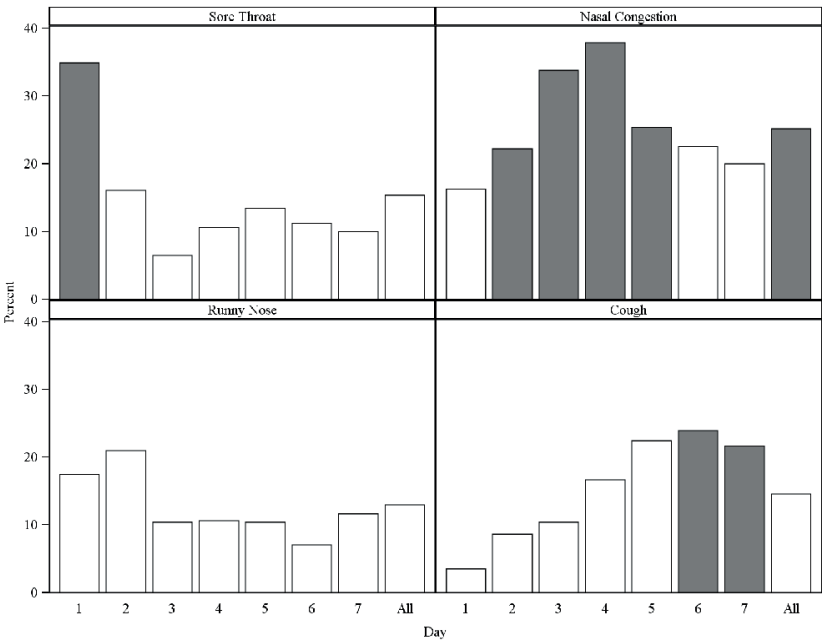

Figure 3. Single most bothersome symptom during a cold. Subjects were asked to report their single "most bothersome" symptom each morning (9 AM) during the first 7 days of their cold. Data are presented as the percent of subjects reporting a particular cold symptom as most bothersome on each day. Shaded bars indicate the symptom receiving the greatest response as the "most bothersome" on a given day of a cold.

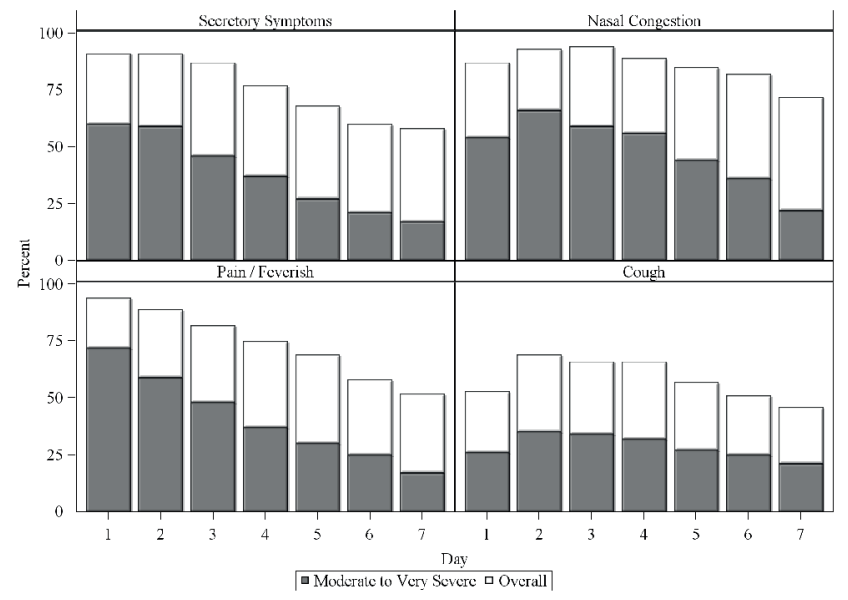

Figure 4. Severity profile of symptoms when grouped by symptom type. The percent of subjects who self-reported one or more symptoms within the symptom group (Overall; open blocks) and symptoms of moderate, severe, or very severe intensity (filled blocks). Secretory symptoms include: runny nose, sneezing and watery eyes. Pain/feverish/inflammatory symptoms include: sore/scratchy throat, headache, muscle aches and pains, feverishness and chilliness. Nasal congestion and cough are represented as individual symptoms. was the "most bothersome" each day (Figure 3). On Day 1, sore throat was reported as the single most bothersome symptom in approximately $35 \%$ of colds, followed by runny nose (17\%) and nasal congestion (16\%). On Days 2-5 of a cold, nasal congestion was reported as the most bothersome symptom with the peak response (38\%) occurring on Day 4. On Day 6 and 7, cough was reported as the most bothersome symptom (24\% Day 6 and $22 \%$ Day 7). Over the entire 7 days, the most bothersome symptom was nasal congestion, followed by sore throat and cough. The single most bothersome symptom of a cold selected on each day at $9 \mathrm{AM}$ tracked closely to the most severe symptom (Figure 2B).

Given the overlap in symptom presentation over the course of cold episodes, it was of interest to group symptom data based on their presumed pathophysiology. We combined symptoms into 4 groups: secretory symptoms (runny nose, sneezing, watery eyes), pain and feverish symptoms (sore/scratchy throat, headache, muscle aches and pains, feverishness and chilliness), nasal congestion and cough. We examined symptom frequency and severity with respect to the four symptom groupings (Figure 4). This categorization revealed that secretory and pain/ feverish symptoms steadily declined from day 1 (Figure 4, open blocks) and this trend was also observed in those reporting moderate-to-severe symptoms (Figure 4, filled blocks). However, a high percentage of subjects reported nasal congestion throughout the week and was considered at least moderate by a majority of subjects on each of the first 4 days of a cold. While less frequent, cough increased on Day 2 and was persistent over the week, even for subjects reporting moderate to very severe cough.

Finally, we examined the chronological relationship between these 4 groups. Table 3 shows the rank order of all possible combinations of these symptom groups and revealed that regardless of the symptoms grouped, the rank order over the entire week was similar to the rank order for each of the 7 days. This observation supports the contention that symptom complex progression occurs in a predictable and continuous manner reflective of the changes in the underlying pathophysiology. Secretory symptoms and nasal congestion were reported together most frequently, followed by nasal congestion and pain/feverish symptoms, secretory and pain/feverish symptoms and secretory, pain/feverish and nasal congestion symptoms. Each of these groups were present at $\geq 60 \%$ over the first 4 days of the cold. Remarkably, $44 \%$ of cold episodes were characterized by the presence of all four symptom groups on Day 1. This association peaked on Day 2 (58\% of cold episodes) and remained around $50 \%$ for each of the first 4 days of a cold.

\section{Discussion}

Studies attempting to characterize the time-course of cold symptoms occurring during natural, or community-acquired colds are challenging for a variety of reasons including difficulty 
Table 3. Summary of two or more symptom groups present at 9 AM (all values in percent of illness episodes).

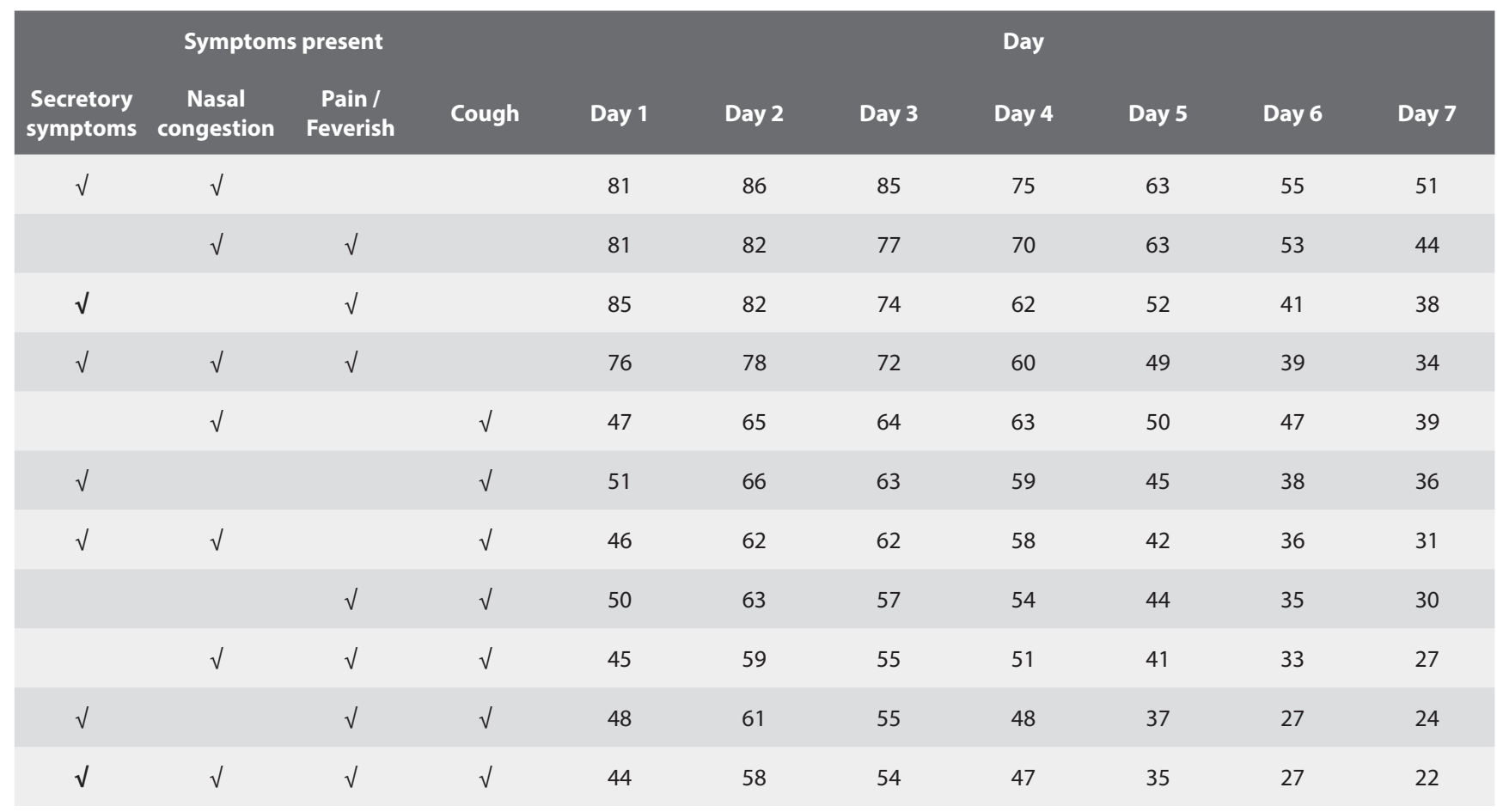

Key cold symptoms are ranked highest to lowest based on total symptom frequency over the week. Key colds symptoms are grouped according to their proximate pathophysiology including secretory symptoms (runny nose, sneezing, watery eyes), pain and feverish symptoms (sore/scratchy throat, headache, muscle aches and pains, feverishness and chilliness), and separately nasal congestion and cough. Percent occurrence, or prevalence, of possible symptom group combinations of two or more symptom groups are listed by day.

in recruiting subjects early in the illness, the need or desire to enroll cold sufferers throughout the year, difficulties in ensuring subjects report symptoms in an accurate and timely manner and the potential influence of compensation incentives, leading investigators to develop and rely on experimental infection models ${ }^{(17)}$. While these models are useful, they usually enroll healthy individuals and are specific to a particular serotype of rhinovirus (e.g. RV-16). In this study we sought to follow the evolution of several key common cold symptoms in self-reported, community-acquired colds collected over a 4 year period. We prospectively enrolled cold sufferers at the workplace which successfully included subjects very early in the cold $(73.5 \%$ enrolled within $24 \mathrm{hrs} ; 87.2 \%$ within $36 \mathrm{hrs}$ ). This strategy also allowed cold episodes to be closely followed over a 4 year period which would include eight rhinovirus-dominant seasons (spring, fall) and colds due to various viral types. Since subjects were already reporting to the workplace and the symptoms were assessed on site at $9 \mathrm{AM}$, this had the potential to ensure accurate and timely reporting of symptoms. Since the subjects were not compensated in relation to the number of colds, or for the duration of reporting symptoms, there was no reason for subjects to report more severe symptoms (in an attempt to be enrolled), or less severe symptoms (in an effort to finish the study quickly).
Investigators sometimes describe the symptoms of the common cold as "staged", for example, an acute (1-2 day) period of sore throat, possibly headache, fatigue, malaise and muscle aches (Days 1-7), followed by a period of nasal congestion, runny nose (Days 2-6) and cough (after Day 2) ${ }^{(19,20)}$. In contrast, our study demonstrates that symptoms pathognomonic of the common cold were described by sufferers as relatively constant in their frequency and severity throughout the day and demonstrated significant overlap in the occurrence of core cold symptoms over the first 7 days of the illness. The most prevalent symptoms overall were nasal congestion and runny nose with sore throat typically a harbinger of the illness. While our study did not extend symptom observations beyond 7 days it is of note that cough severity exhibited a somewhat slower decline than other symptoms late in the cold, particularly more severe cough (Figure 4), consistent with the likelihood of a continuing post-infectious cough. In the case of rhinovirus infection, the predominance of sore throat early on is likely a result of its binding to ICAM-1, which is constitutively expressed in the posterior pharynx (21) and the immune response against it mounted within the NALT (nasal associated lymphoid tissue). The symptoms of runny nose and nasal congestion may be due to the posterior spread of the virus into the nasal passageways. 
Unique to this study was the assessment of the single "most bothersome" cold symptom in an attempt to determine which symptom they perceived as having the biggest impact on his/ her daily well-being. This assessment (in approximately $40 \%$ of cold episodes) revealed that the single most bothersome symptom was sore/scratchy throat on Day 1 , followed by nasal congestion from Days 2-5, and finally cough on Days 6-7. These outcomes confirmed the progression of the subjective symptom responses given by the subjects as it forced them to attest to the single most bothersome symptom each day---thus, the progression from sore throat to nasal congestion to cough. Many reviews of the common cold describe a more linear, simplistic symptom progression, while our data demonstrate significant overlap in the appearance of symptoms. This difference could be attributed, in part, to our enrollment of subjects very early in the cold, symptoms reported from induced cold studies and/or subjects focusing more on their 'most bothersome' symptoms in surveys or compensated studies. This latter point is interesting as the traditional progression of symptoms (sore throat, nasal congestion, cough) is more similar to the most bothersome data reported in the current study.

One perspective that may obscure symptom progression, in part, is that each symptom evaluated may have a varying, or changing, etiology. For example, cough and sore throat subsume several possible proximate causes while nasal congestion and runny nose may not. This effect may explain some of the variance in how symptoms evolve over time. For example, a dry, scratchy throat often signals the start of a cold while sore throat later in the cold may be a consequence of other processes (e.g., post-nasal drip). Likewise, cough early in a cold may be caused by post-nasal drip, or throat clearing, while cough late in the cold may signal lower airway involvement, for example, due to airway hyperreactivity and/or viral expansion in the lower airway. In addition, recent work by Abdullah ${ }^{(22)}$ has suggested that rhinovirus infection in cultured human neuronal cells enhances the expression of transient receptor potential (TRP) channels (eg, capsaicin sensitive TRPV-1), which have been implicated as putative cough receptors. It is possible that sufficient expression of these receptors in vivo may escalate over the course of a cold and contribute to the increased cough sensitivity observed in subjects with URTI ${ }^{(23,24)}$.

The patterns of symptoms in our natural colds study are different than those described originally by Jackson (1958) in which runny nose and sore throat predominated early on while nasal congestion and cough were not considered major symptoms. The difference between our study and the investigation by Jackson could be due to the latter being a viral challenge study in which symptoms are typically milder ${ }^{(20)}$. Our study found that subjects reported a high degree of overlap in daily symptoms, underscoring the multi-symptomatic nature of colds which may explain the popularity of fixed-dose combination cough, cold and flu products. Surprisingly, about half the subjects experienced concurrent symptoms from the 4 symptom groupings during the first 4 days and over $20 \%$ still experiencing symptoms at Day 7. Given that a recent global survey found that $73 \%$ of cold sufferers mostly rely on a physician or pharmacist to assist in selecting appropriate medication for palliative care of symptoms, knowledge of the symptomatology of the cold syndrome amongst medical professionals is worthwhile ${ }^{(25)}$.

This study was not without its limitations. For one, enrolling subjects at the work site is problematic since subjects with very severe illness may choose not to attend work and thus may be excluded from the dataset. Additionally, while subjects at entry were instructed to refrain from taking medications to treat their cold symptoms, subjects may have taken a dose of OTC medication to treat their symptoms in the absence of constant surveillance. However, if this occurred, it would only serve to under-report the severity of symptoms and would unlikely effect symptom frequency. The number of yearly cold episodes reported during the surveillance period was $<1$ /year, which is lower than found in the literature. This is not overly surprising given the stringent entry criterion ( $<48$ hours of symptom onset), required daily reporting during the weekdays (9 AM), subjects not reporting to work and exclusion of those cold episodes resulting in physician intervention.

In conclusion, this prospective natural history study characterizes the symptomatology of 226 community-acquired colds beginning at the earliest stage of the illness. The most surprising finding was the daily coexistence of symptoms, which has not been previously emphasized in either community-acquired, or induced-cold studies. Sore throat, nasal congestion and runny nose were prevalent and impactful. Although cough was not as frequent as other symptoms (eg, nasal congestion, runny nose, sore throat), when it was present, it was considered particularly bothersome. These data provide a novel assessment of symptom frequency and severity of 226 cold episodes and serves as a reasonable reference for prediction of daily symptom burden that might be experienced by a typical common cold sufferer. This information may assist in the understanding of the pathophysiology of the cold and inform appropriate treatment selection.

\section{Acknowledgement}

The authors thank Felicia Coates for her assistance in the virology and clinical laboratory. We acknowledge the participation and informed consent of Richardson-Vicks, Inc. employees. Richardson-Vicks, Inc., a subsidiary of Procter \& Gamble, sponsored the trial as part of a commitment to basic research into the common cold. 


\section{Authorship contribution}

DKR and TW conceived, designed and executed the clinical protocol. DKR, TW, DR, and AC analyzed and interpreted the data. DKR, AC, DR and TW wrote the manuscript.

\section{Disclaimers}

DR and AC are currently full-time employees of the Procter \& Gamble Company. At the time the study was conducted, TW and DKR were full-time employees of Richardson-Vicks, Inc., a subsidiary of the Procter \& Gamble Company. At the time of manscript writing, TW was an employee of Boehringer-Ingelheim Pharmaceuticals, Inc., Ridgefield, CT 06877, USA. DKR is currently President \& Founder, On Point Advisors, LLC, PO Box 5450, Chattanooga, TN 37406, USA. The in-life period of this study occurred between 1987 and 1992.

Portions of this work have been previously published as meeting abstracts: Cohen SD, Witek TJ, Geist FC, Sorrentino JV and Riker DK. 1992, The clinical epidemiology of acute upper respiratory infection, XIV Congress of the European Rhinological Society, Abstract, A111.

"Towards effective patient management and care in the pharmacy", In Common Cold Study Forum. edited by R. Eccles, 1993 Royal Society of Medicine Services International Congress and Symposium Series No. 206, published by Royal Society of Medicine Services, Ltd.

This research was not obligated to be registered in a public clinical database, nor was this an interventional clinical trial using any drug, or drug product, requiring retrospective registration.

\section{Conflicts of Interest}

All authors have been employed by the sponsor at various times. However, no commercial products were tested, nor are branded products mentioned, or recommended. DKR and TW are not employees of nor do they have any financial relationships with Procter \& Gamble.

\section{References}

1. Nichol K, Heilly S and Ehlinger E. Colds and influenza-like illnesses in university students: impact on health, academic and work performance, and health care use. Clin Infect Dis. 2005; 40: 1263-1270.

2. Fendrick A, Monto A, Nightengale B, Sarnes $M$. The economic burden of non-influenzarelated viral respiratory tract infection in the United States. Arch Intern Med. 2003; 163: 487-494.

3. Turner R. Rhinovirus: more than just a common cold virus. J Infect Dis. 2007; 195: 765 766.

4. Turner R. Epidemiology, pathogenesis, and treatment of the common cold. Ann Allergy Asthma Immunol. 1997; 78: 531-539.

5. Heikkinen T, Järvinen A. The Common Cold Lancet. 2003; 361: 51-59.

6. Eccles R. Efficacy and safety of over-thecounter analgesics in the treatment of common cold and flu. J Clin Pharmacy Thera. 2006: 31: 309-319.

7. Vernacchio L, Kelly J, Kaufman D, Mitchell A. Cough and cold medication use by US children, 1999-2006: results from the Slone survey. Pediatrics. 2008; 122: 323-329.

8. Monto, A. and Ullman, B. Acute respiratory illness in an American community. JAMA 1974: 227: 164-169.

9. Winther B, Gwaltney Jr J, Mygind N, Turner $\mathrm{R}$, Hendley J. Sites of rhinovirus recovery after point inoculation of the upper airway. JAMA 1986; 256 (13): 1763-1767.

10. Gwaltney Jr J, Hendley J, Simon G, Jordan $\mathrm{Jr}$ W. Rhinovirus infections in an industrial population. JAMA. 1967; 202: 494-500

11. Turner R, Witek T, Riker D. Comparison of symptom severity in natural and experimentally induced colds. Amer J Rhinol. 1996; 10: 167-172.

12. Arruda E, Pitkäranta A, Witek T, Doyle C Hayden F. Frequency and natural history of rhinovirus infections in adults during autumn. J Clin Micro. 1997; 35: 2864-2868.

13. Tyrrell D, Cohen S, Schilarb J. Signs and symptoms in common colds. Epi Infect. 1993: 111: 143-156.

14. Gwaltney JM, Hendley O, Simon G, Jordan Jr WS. Rhinovirus infections in an industrial population. JAMA. 1967, 202: 494-500.

15. Barrett B, Brown R, Mundt M, et al. The Wisconsin upper respiratory symptom survey is responsive, reliable, and valid. J Clin Epi. 2005; 58: 609-617.

16. Jackson GG, Dowling HF, Spiesman, IG, Boand, AV. Transmission of the common cold to volunteers under controlled conditions. I. The common cold as a clinical entity. AMA Arch Intern Med.1958; 101: 267-78.

17. Gwaltney J, Hendley J, Patrie J. Symptom severity patterns in experimental common colds and their usefulness in timing onset of illness in natural colds. Clin Infect Dis. 2003; 36: 714-723.

18. Smith A, Tyrrell D, Coyle K, Higgins $P$, Willman J. Diurnal variation in the symptoms of colds and influenza. Chronobiol Int. 1988; 5: 411-416.

19. Jackson Allen P, Simenson S. Management of common cold symptoms with over-thecounter medications: clearing the confusion. Postgrad Med. 2013; 125: 73-81.

20. Eccles R. Is the common cold a clinical entity or a cultural concept? Rhinology. 2013, 51:3-8.
21. Winther B, Kawana R, Saito H. Fireside conference 11. Common cold. Rhinol Suppl. 1992; 14: 228-232

22. Abdullah H, Heaney LG, Cosby SL, McGarvey LP. Rhinovirus upregulates transient receptor potential channels in a human neuronal cell line: implications for respiratory virus-induced cough reflex sensitivity. Thorax. 2014; 69: 46-54.

23. Dicpinigaitis $P V$, Bhat $R$, Rhoton WA, Tibb AS, Negassa A. Effect of viral upper respiratory tract infection on the urge-to-cough sensation. Respir Med. 2011; 105: 615-618.

24. O'Connell F, Thomas VE, Studham JM, Pride NB, Fuller RW. Capsaicin cough sensitivity increases during upper respiratory infection. Respir Med. 1996; 90: 279-286.

25. Hull JD, Barton IP, Torgersen J, McNeil, CM, A Survey of the Experience and Impact of Acute Upper Respiratory Tract Infections on People in Six Countries in the 2011/2012 Common Cold and Flu Season. Open J Resp Dis. 2013; 3: 175-187.

Andrew N. Carr, PhD

Procter \& Gamble

8700 Mason Montgomery Rd.

Mason $\mathrm{OH} 45040$

USA

Tel: +1-513-622 0928

E-mail:carr.an@pg.com 